\title{
Acidentes e violências entre mulheres atendidas em Serviços de Emergência Sentinela - Brasil, 2009
}

\author{
Accidents and violence among women \\ attended in Sentinel Emergency Services - Brazil, 2009
}

\author{
Celeste de Souza Rodrigues ${ }^{1}$ \\ Deborah Carvalho Malta ${ }^{2}$ \\ Tatau Godinho ${ }^{3}$ \\ Márcio Dênis Medeiros Mascarenhas ${ }^{2}$ \\ Marta Maria Alves da Silva ${ }^{2}$ \\ Rurany Ester Silva ${ }^{2}$
}

${ }^{1}$ Secretaria de Atenção à Saúde, Ministério da Saúde. Esplanada dos Ministérios Bloco G/90. 70058-900

Brasília DF.

celeste.rodrigues@saude.gov.br

2 Secretaria de Vigilância à

Saúde, Ministério da Saúde

${ }^{3}$ Secretaria Especial de

Políticas para Mulheres

\begin{abstract}
Accidents from external causes affect the human population in different ways. This article seeks to analyze emergency care for women who are victims of accidents and violence. Data from the Surveillance System for Violence and Accidents were analyzed. This study was carried out in 74 emergency units of 23 state capitals and the Federal District in 2009 and included 6,965 women aged from 20-59 years. The age groups of 20-39 and 40-59 years were compared for the occurrence of accidents and violence. Accidents were more frequent among young black women (20-39 years) with more than nine years of schooling. The occurrence of violence was also prevalent in young black women but with less schooling. Falls were the most frequent accidents (38.6\%), followed by traffic accidents. The occurrence of violence was more frequent in the home $(p<0.000)$ and the mention of alcohol abuse among victims of violence was predominant. The most frequent type of violence was aggression (84.6\%), in which the aggressor was male (79.1\%) and identified as an intimate partner (44.1\%). It is increasingly important that services are able to provide comprehensive and humanized care to the victims of this important public health problem.
\end{abstract}

Key words Violence against women, External causes, Violence, Accidents, Emergency medical care, Epidemiology
Resumo As causas externas afetam de maneira desigual as populações humanas. O presente artigo tem como objetivo analisar os atendimentos de emergência em mulheres vítimas de acidentes e violências. Foram analisados dados do inquérito de Vigilância de Violências e Acidentes em 74 Unidades de Emergência de 23 capitais e no Distrito Federal em 2009. Analisaram-se 6.965 atendimentos de mulheres adultas comparando-se as faixas de 20-39 e 40-59 anos, em relação a ocorrência de acidentes e violências. Os acidentes foram mais frequentes em mulheres jovens (20 a 39 anos), de cor negra e com escolaridade maior que 9 anos de estudo. A ocorrência de violência também foi predominante em mulheres jovens e negras, porém com menor escolaridade. Entre os acidentes predominaram as quedas (38,6\%), seguidas de acidentes de transporte. As violências foram mais frequentes no domicílio $(p<0,000) e$ a referencia ao uso de álcool predominou entre as vítimas de violência. O tipo de violência mais frequente foi a agressão (84,6\%), sendo o agressor do sexo masculino $(79,1 \%)$ e identificado como parceiro intimo em $44,1 \%$. É cada vez mais relevante que os serviços estejam capacitados para a atenção integral e humanizada às vítimas desse importante problema de saúde pública.

Palavras-chave Violência contra a mulher, Causas externas, Violência, Acidentes, Serviços de emergência, Epidemiologia 


\section{Introdução}

As causas externas (acidentes e violências) são responsáveis por um significativo número de óbitos e sequelas, trazendo graves prejuízos ao indivíduo, às famílias e à sociedade. Apresentando tendência crescente nas últimas décadas, as causas externas constituem-se em importante problema de saúde pública trazendo inquietação aos gestores $^{1}$. No Brasil, em 2009, representavam a terceira causa de morte na população geral e a primeira na população de 1 a 39 anos de idade ${ }^{1}$.

O Ministério da Saúde (MS), considerando a relevância do tema e a necessidade de orientar a atuação do setor saúde, elaborou a "Política Nacional de Redução da Morbimortalidade por Acidentes e Violências"', dando espaço a novos debates na agenda da saúde. Nesta política uma das prioridades é a implementação de ações de vigilância e monitoramento dos acidentes e das violências.

Buscando desenvolver esse monitoramento e considerando as limitações dos sistemas de informação existentes até então, que registravam e descreviam apenas os casos de acidentes e violências cujo desfecho havia sido a internação ou o óbito, o MS implantou, em 2006, a Vigilância de Violências e Acidentes em Serviços Sentinela de Urgência e Emergência (VIVA Inquérito), que é um dos componentes do Sistema de Vigilância de Violências e Acidentes (VIVA), para a obtenção de dados que possibilitassem o conhecimento da real dimensão do problema ${ }^{3}$. Além da ampliação e qualificação dos dados, a vigilância permite a identificação de fatores de risco e proteção relacionados aos casos de acidentes e violências, o que pode ser fundamental para dimensionar a magnitude do problema e direcionar a (re)organização dos serviços de saúde com o desenvolvimento de ações de atenção e proteção às vítimas, de prevenção das ocorrências e de promoção da saúde e da cultura de paz.

Estudos mostram que os acidentes e a violência não afetam a população de maneira uniforme, o risco varia de acordo com o sexo, raça/ cor, idade e condição socioeconômica ${ }^{4-6}$. Dados do VIVA nos biênios 2006/20077 e 2008/20098 confirmam essa diferença apontando a importância do aprofundamento da análise e do conhecimento do comportamento e da dimensão desses eventos em segmentos específicos da população. Schraiber et al. ${ }^{6}$ discutem a violência também como uma questão de gênero representando diferenças de poder e desigualdades de valor social.

As ocorrências de causas externas, acidentes e violências, são mais frequentes entre os homens e por isto existe maior documentação e estudos junto a população masculina ${ }^{7,8}$. O tema da violência contra a mulher vem sendo analisado em estudos nacionais e globais ${ }^{6,9}$, mas ainda existem lacunas de conhecimento devido a sua natureza que envolve a complexa trama da violência doméstica, quando a mesma é praticada por parceiros íntimos, resultando na maioria das vezes no ocultamento das relações de opressão e na repetição dos atos violentos, nas suas mais diversas expressões. O registro destas ocorrências nas unidades de atendimento de Emergência Sentinela para causas externas contribui na compreensão destas situações e no apoio às politicas públicas de prevenção e promoção à saúde.

Assim, o estudo proposto pretende analisar os dados referentes ao Inquérito VIVA 2009 no que se refere às mulheres vítimas de acidentes e violências, buscando agregar questões relevantes à discussão de gênero no âmbito das causas externas.

\section{Métodos}

O Inquérito VIVA 2009 foi realizado em 74 Unidades de Emergência distribuídas em 23 capitais e no Distrito Federal. Estimou-se um tamanho mínimo de entrevistas referentes a 1.500 atendimentos por causas externas (acidentes e violências) em cada uma das capitais. Foi utilizado processo de amostragem por conglomerado em único estágio, sendo o turno de trabalho de 12 horas a unidade primária de amostragem (UPA). Os turnos de atendimento dos serviços foram sorteados, dentre os 60 turnos possíveis durante o mês de setembro de 2009. Para a definição do número de turnos pesquisados tomou-se em conta o tamanho mínimo da amostra de atendimentos por causas externas no mesmo estabelecimento ocorrido em anos anteriores. A metodologia de seleção dos estabelecimentos está descrita em outra publicação ${ }^{8}$.

Os coordenadores de campo dos estados e municípios foram capacitados pela equipe técnica do Ministério da Saúde (MS), para a padronização dos procedimentos da pesquisa. Foram utilizadas fichas padronizadas, testadas em inquéritos prévios. O informante era a vítima, os pais ou acompanhantes, quando o paciente era uma criança ou encontrava-se impossibilitado de responder.

O projeto foi aprovado pela Comissão Nacional de Ética em Pesquisa (Conep), atendendo às recomendações da Resolução 196/ $1996^{10}$. Como os dados são utilizados para fins 
de vigilância epidemiológica, a assinatura do termo de consentimento livre e esclarecido foi substituída por consentimento verbal, obtido pelo paciente ou por seu responsável. Foi garantido total anonimato e privacidade aos pacientes, acompanhantes e profissionais, bem como a liberdade para desistir da entrevista a qualquer momento.

Os dados foram digitados nos municípios e transferidos para o MS para análise de consistência e duplicidade, utilizando o programa Link Plus, versão 2.0. As análises foram processadas no programa STATA versão $10^{11}$.

Foram analisadas as causas externas (acidentes e violências), segundo as seguintes variáveis: sexo, faixa etária, raça/cor, escolaridade, presença de deficiência, ocorrência no domicilio, uso de bebida alcoólica pela vítima, evento relacionado ao trabalho, natureza do evento, se teve atendimento prévio, período de atendimento, dia de atendimento, locomoção para o hospital, natureza da lesão, parte do corpo atingida e evolução na emergência. No caso das vítimas de acidentes foram analisadas as variáveis referentes ao tipo de acidente (acidente de transporte - AT, quedas, queimadura, outros acidentes), tipo de vítima de AT (pedestre, condutor, passageiro), tipo de meio de locomoção (a pé, automóvel, motocicleta, bicicleta, transporte coletivo), tipo de queda, tipo de queimadura, outros acidentes. No caso de violência, foram descritos os tipos (autoprovocada ou interpessoal/maus tratos/agressão), caracterização do agressor (relação com a vítima, sexo), natureza das violências (física, sexual, psicológica, negligência/abandono).

Para este estudo foram selecionadas as mulheres adultas (faixa etária de 20 a 59 anos). As variáveis foram comparadas segundo a sua ocorrência e analisadas segundo os grupos etários de 20 a 39 anos e 40 a 59 anos, testando-se a hipótese nula de independência entre as variáveis qualitativas. Foi utilizado o teste qui-quadrado, com nível de significância de 5\%.

\section{Resultados}

Foram analisadas informações referentes a 6.965 mulheres de 20 a 59 anos participantes do Inquérito VIVA 2009, sendo 6.248 (89,7\%) vítimas de acidentes e 717 (10,3\%) vítimas de violências.

As características das mulheres atendidas nos serviços de emergência, segundo o tipo de ocorrência, estão apresentadas na Tabela 1. Em relação à idade, 63,7\% tinham entre 20 e 39 anos, e nesta faixa etária foi maior a proporção de violências $(74,6 \% ; \mathrm{p}=0,0000)$. Quanto à raça/cor, $59,9 \%$ eram mulheres negras (parda e preta). Mulheres de cor preta e parda tiveram maior frequência de violência $71,7 \%(p=0,0000)$. Os acidentes foram mais frequentes em mulheres de escolaridade igual ou maior que 9 anos (54,8\%), sendo que a violência foi mais frequente entre as de menor escolaridade $(54,7 \% ; \mathrm{p}=0,0000)$. A existência de algum tipo de deficiência foi observada em 4\% das mulheres e 9\% declararam uso de bebida alcóolica, sendo maior a proporção nos casos de violência, $28,6 \%(\mathrm{p}=0,0000)$. A ocorrência no domicilio foi mais frequente nos casos de violência $(59,6 \% ; \mathrm{p}=0,0000)$ e os eventos relacionados ao trabalho foram mais frequentes nos casos de acidentes $(25,1 \% ; \mathrm{p}=0,000)$. Dentre os acidentes, a maioria dos eventos foram considerados acidentais (93,3\%; $\mathrm{p}=0,0000)$. Foram atendidos em outro serviço previamente $18,4 \%$ das mulheres. O modo de locomoção mais frequente para o hospital foi em veículo particular, seguido pelo transporte coletivo/a pé e pelo atendimento pré-hospitalar (Serviço de Atendimento Móvel de Urgência - SAMU). Em relação à natureza da lesão predominaram os cortes/laceração nos atendimentos decorrentes de violências e as contusões/entorses/luxação naqueles em consequência de acidentes $(p=0,0000)$. A cabeça/pescoço foi a parte mais frequentemente atingida em violências e os membros inferiores em acidentes $(\mathrm{p}=0,0000)$. A alta foi o desfecho prevalente, em $82,6 \%$ dos casos de acidentes e em $79,3 \%$ daqueles relacionados às violências; a internação ocorreu em 10,3\% dos atendimentos por violências $(\mathrm{p}=0,0035)$.

Na Tabela 2 é mostrada a distribuição dos acidentes e violências segundo faixa etária do estudo. Os acidentes foram mais frequentes em mulheres mais jovens (20 a 39 anos) e negras (preta/parda) $(\mathrm{p}=0,0342)$, com 9 anos ou mais de estudo ( $p=0,0000)$; enquanto a violência atingiu mais mulheres de menor escolaridade sem diferença estatisticamente significativa quanto a faixa etária. Mulheres de 40 a 59 anos sofreram mais acidentes no domicílio em relação às mais jovens $(\mathrm{p}=0,0000)$. O uso do álcool foi declarado em maior proporção pelas vítimas de violência, não sendo observada diferença segundo faixa etária. Nos casos de acidentes, o uso do álcool foi declarado principalmente pelas mulheres mais jovens $(\mathrm{p}=0,0000)$. Nos casos de acidentes o modo de locomoção mais frequente para o hospital foi o veículo particular em ambas as faixas etárias, todavia mulheres de 20 a 39 anos usaram 
Tabela 1. Distribuição dos atendimentos de emergência por acidentes e violência entre mulheres adultas segundo variáveis selecionadas e tipo de ocorrência, em 23 capitais e Distrito Federal, Brasil, 2009.

\begin{tabular}{|c|c|c|c|c|}
\hline \multirow[b]{2}{*}{ Características } & \multicolumn{4}{|c|}{ Tipo de ocorrência ${ }^{a}$} \\
\hline & $\begin{array}{c}\text { Total } \\
(\mathrm{n}=6.965) \\
\%\end{array}$ & $\begin{array}{c}\text { Acidentes } \\
(\mathrm{n}=6.248) \\
\%\end{array}$ & $\begin{array}{c}\text { Violência } \\
(\mathbf{n}=717) \\
\%\end{array}$ & Valor de $\mathrm{p}^{\mathrm{b}}$ \\
\hline \multicolumn{5}{|l|}{ Faixa etária (anos) } \\
\hline 20 a 39 & 63,7 & 62,5 & 74,6 & 0,0000 \\
\hline 40 a 59 & 36,3 & 37,5 & 25,4 & \\
\hline \multicolumn{5}{|l|}{ Raça/cor } \\
\hline Branca & 37,3 & 38,7 & 25,0 & 0,0000 \\
\hline Preta/parda & 59,9 & 58,6 & 71,7 & \\
\hline Amarela/indígena & 2,8 & 2,8 & 3,3 & \\
\hline \multicolumn{5}{|l|}{ Escolaridade (anos de estudo) } \\
\hline 0 a 8 & 46,1 & 45,2 & 54,7 & 0,0000 \\
\hline $9 \mathrm{e}+$ & 54,0 & 54,8 & 45,3 & \\
\hline \multicolumn{5}{|l|}{ Possui algum tipo de deficiência ${ }^{c}$} \\
\hline Sim & 4,0 & 4,0 & 3,6 & 0,6393 \\
\hline Não & 96,0 & 96,0 & 96,4 & \\
\hline \multicolumn{5}{|l|}{ Ocorrência no domicílio } \\
\hline Sim & 47,3 & 46,0 & 59,6 & 0,0000 \\
\hline Não & 52,7 & 54,1 & 40,4 & \\
\hline \multicolumn{5}{|l|}{ Declaração de uso de álcool pela vítima } \\
\hline Sim & 9,0 & 6,6 & 28,6 & 0,0000 \\
\hline Não & 91,0 & 93,4 & 71,4 & \\
\hline \multicolumn{5}{|l|}{ Evento relacionado ao trabalho } \\
\hline Sim & 23,4 & 25,1 & 8,2 & 0,0000 \\
\hline Não & 76,6 & 74,9 & 91,8 & \\
\hline \multicolumn{5}{|l|}{ Evento considerado acidental } \\
\hline Sim & 85,5 & 93,3 & 15,0 & 0,0000 \\
\hline Não & 14,5 & 6,7 & 85,0 & \\
\hline \multicolumn{5}{|l|}{ Atendimento prévio em outro serviço } \\
\hline Sim & 18,4 & 18,1 & 21,2 & 0,1955 \\
\hline Não & 81,6 & 81,9 & 78,8 & \\
\hline \multicolumn{5}{|l|}{ Período do atendimento } \\
\hline Diurno (07h00min-18h59min) & 62,6 & 64,7 & 44,0 & 0,0000 \\
\hline Noturno (19h00min-06h59min) & 37,4 & 35,3 & 56,0 & \\
\hline \multicolumn{5}{|l|}{ Dia do atendimento } \\
\hline Sábado e Domingo & 33,0 & 31,9 & 42,8 & 0,0001 \\
\hline Segunda a Sexta & 67,0 & 68,1 & 57,2 & \\
\hline \multicolumn{5}{|l|}{ Locomoção para o hospital } \\
\hline A pé/transporte coletivo & 27,7 & 28,1 & 24,6 & 0,0000 \\
\hline Veículo particular & 47,4 & 48,3 & 39,3 & \\
\hline Assistência pré-hospitalar ${ }^{d}$ & 22,0 & 21,9 & 22,3 & \\
\hline Outros $^{\mathrm{e}}$ & 2,9 & 1,7 & 13,8 & \\
\hline \multicolumn{5}{|l|}{ Natureza da lesão } \\
\hline Contusão/entorse/luxação & 49,6 & 51,7 & 30,0 & 0,0000 \\
\hline Corte/laceração/amputação & 25,5 & 23,5 & 44,0 & \\
\hline Fratura/trauma ${ }^{\mathrm{f}}$ & 16,6 & 17,0 & 12,7 & \\
\hline Outros $^{g}$ & 8,3 & 7,8 & 13,4 & \\
\hline \multicolumn{5}{|l|}{ Parte do corpo atingida } \\
\hline Cabeça/pescoço & 16,5 & 14,1 & 39,8 & 0,0000 \\
\hline Tórax/abdome/pélvis & 10,1 & 10,0 & 10,3 & \\
\hline Membros superiores & 26,4 & 26,6 & 24,9 & \\
\hline Membros inferiores & 38,5 & 41,6 & 8,2 & \\
\hline Múltiplos órgãos/regiões & 8,5 & 7,6 & 16,9 & \\
\hline \multicolumn{5}{|l|}{ Evoluçãa $\mathrm{O}^{\mathrm{h}}$} \\
\hline Alta & 82,3 & 82,6 & 79,3 & 0,0035 \\
\hline Internação hospitalar & 6,9 & 6,5 & 10,3 & \\
\hline Transferência para outro serviço & 10,9 & 11,0 & 10,4 & \\
\hline
\end{tabular}

Fonte: MS, SVS, Sistema de Vigilância de Violências e Acidentes-VIVA, Inquérito 2009. a) Para algumas variáveis o número de atendimentos divergiu devido a dados faltantes (ignorado/em branco). b) Teste do qui-quadrado. c) Inclui deficiência física, mental, visual, auditiva, outras deficiências/síndromes. d) Inclui unidades do Serviço de Atendimento Móvel de Urgência (SAMU), ambulâncias, resgate. e) Inclui viatura policial e outros. f) Inclui trauma cranioencefálico, trauma dentário, politraumatismo. g) Inclui intoxicação/envenenamento, queimadura, outros. h) Excluídos os casos que evoluíram ao óbito ( $\mathrm{n}=$ 8) nas primeiras 24 horas de atendimento. 
Tabela 2. Distribuição dos atendimentos de emergência por acidentes e violência entre mulheres adultas segundo faixa etária. 23 capitais e Distrito Federal, Brasil, 2009.

\begin{tabular}{|c|c|c|c|c|}
\hline \multirow[b]{2}{*}{ Características } & \multicolumn{3}{|c|}{ Acidentes $^{\mathrm{a}}$} & \multirow[b]{2}{*}{ Valor de $\mathrm{p}^{\mathrm{t}}$} \\
\hline & $\begin{array}{c}\text { Total } \\
(\mathrm{n}=6.179) \\
\%\end{array}$ & $\begin{array}{c}20-39 \\
(\mathrm{n}=3.958) \\
\%\end{array}$ & $\begin{array}{c}40-59 \\
(\mathrm{n}=2.221) \\
\%\end{array}$ & \\
\hline \multicolumn{5}{|l|}{ Raca/cor } \\
\hline Branca & 38,7 & 37,3 & 41,0 & 0,0363 \\
\hline Preta/parda & 58,6 & 59,7 & 56,7 & \\
\hline Amarela/indígena & 2,8 & 3,0 & 2,3 & \\
\hline \multicolumn{5}{|l|}{ Escolaridade (anos de estudo) } \\
\hline 0 a 8 & 45,2 & 35,3 & 61,8 & 0,0000 \\
\hline $9 \mathrm{e}+$ & 54,8 & 64,7 & 38,3 & \\
\hline \multicolumn{5}{|l|}{ Possui algum tipo de deficiência ${ }^{c}$} \\
\hline Sim & 4,0 & 2,1 & 7,4 & 0,0000 \\
\hline Não & 96,0 & 97,9 & 92,7 & \\
\hline \multicolumn{5}{|l|}{ Ocorrência no domicílio } \\
\hline Sim & 46,0 & 40,1 & 55,7 & 0,0000 \\
\hline Não & 54,1 & 59,9 & 44,3 & \\
\hline \multicolumn{5}{|l|}{ Declaração de uso de álcool pela vítima } \\
\hline Sim & 6,6 & 8,1 & 4,3 & 0,0000 \\
\hline Não & 93,4 & 92,0 & 95,7 & \\
\hline \multicolumn{5}{|l|}{ Evento relacionado ao trabalho } \\
\hline Sim & 25,1 & 26,2 & 23,4 & 0,0539 \\
\hline Não & 74,9 & 73,8 & 76,6 & \\
\hline \multicolumn{5}{|l|}{ Evento considerado acidental } \\
\hline Sim & 93,3 & 93,3 & 93,2 & 0,8711 \\
\hline Não & 6,7 & 6,7 & 6,8 & \\
\hline \multicolumn{5}{|l|}{ Atendimento prévio em outro serviço } \\
\hline Sim & 18,1 & 17,4 & 19,2 & 0,154 \\
\hline Não & 81,9 & 82,6 & 80,8 & \\
\hline \multicolumn{5}{|l|}{ Período do atendimento } \\
\hline Diurno (07h00min-18h59min) & 64,7 & 62,3 & 68,7 & 0,0000 \\
\hline Noturno (19h00min-06h59min) & 35,3 & 37,8 & 31,3 & \\
\hline \multicolumn{5}{|l|}{ Dia do atendimento } \\
\hline Sábado e Domingo & 31,9 & 32,7 & 30,5 & 0,1532 \\
\hline Segunda a Sexta & 68,1 & 67,4 & 69,5 & \\
\hline \multicolumn{5}{|l|}{ Locomoção para o hospital } \\
\hline A pé/transporte coletivo & 28,1 & 26,2 & 31,3 & 0,0002 \\
\hline Veículo particular & 48,3 & 48,8 & 47,5 & \\
\hline Assistência pré-hospitalar ${ }^{\mathrm{d}}$ & 21,9 & 23,3 & 19,6 & \\
\hline Outros $^{\mathrm{e}}$ & 1,7 & 1,8 & 1,7 & \\
\hline \multicolumn{5}{|l|}{ Natureza da lesão } \\
\hline Contusão/entorse/luxação & 51,7 & 51,5 & 52,1 & 0,0976 \\
\hline Corte/laceração/amputação & 23,5 & 24,0 & 22,7 & \\
\hline Fratura/trauma ${ }^{\mathrm{f}}$ & 17,0 & 16,2 & 18,3 & \\
\hline Outros ${ }^{\mathrm{g}}$ & 7,8 & 8,3 & 6,8 & \\
\hline \multicolumn{5}{|l|}{ Parte do corpo atingida } \\
\hline Cabeça/pescoço & 14,1 & 13,6 & 15,0 & 0,0013 \\
\hline Tórax/abdome/pélvis & 10,0 & 9,4 & 11,1 & \\
\hline Membros superiores & 26,6 & 26,5 & 26,7 & \\
\hline Membros inferiores & 41,6 & 41,7 & 41,5 & \\
\hline Múltiplos órgãos/regiões & 7,6 & 8,8 & 5,7 & \\
\hline \multicolumn{5}{|l|}{ Evolução $\mathrm{o}^{\mathrm{h}}$} \\
\hline Alta & 82,6 & 82,7 & 82,4 & 0,6091 \\
\hline Internação hospitalar & 6,5 & 6,7 & 6,2 & \\
\hline Transferência para outro serviço & 11,0 & 10,7 & 11,4 & \\
\hline
\end{tabular}


Tabela 2. continuação

\begin{tabular}{|c|c|c|c|c|}
\hline \multirow[b]{2}{*}{ Características } & \multicolumn{3}{|c|}{ Violência $^{a}$} & \multirow[b]{2}{*}{ Valor de $\mathrm{p}^{\mathrm{t}}$} \\
\hline & $\begin{array}{c}\text { Total } \\
(\mathbf{n}=707) \\
\%\end{array}$ & $\begin{array}{c}20-39 \\
(\mathrm{n}=545) \\
\%\end{array}$ & $\begin{array}{c}40-59 \\
(n=162) \\
\%\end{array}$ & \\
\hline \multicolumn{5}{|l|}{ Raça/cor } \\
\hline Branca & 25,0 & 24,1 & 27,9 & 0,5819 \\
\hline Preta/parda & 71,7 & 72,8 & 68,4 & \\
\hline Amarela/indígena & 3,3 & 3,1 & 3,7 & \\
\hline \multicolumn{5}{|l|}{ Escolaridade (anos de estudo) } \\
\hline 0 a 8 & 54,7 & 52,5 & 60,8 & 0,0945 \\
\hline $9 \mathrm{e}+$ & 45,3 & 47,5 & 39,2 & \\
\hline \multicolumn{5}{|l|}{ Possui algum tipo de deficiência ${ }^{c}$} \\
\hline Sim & 3,6 & 3,7 & 3,3 & 0,8141 \\
\hline Não & 96,4 & 96,3 & 96,7 & \\
\hline \multicolumn{5}{|l|}{ Ocorrência no domicílio } \\
\hline Sim & 59,6 & 57,9 & 64,4 & 0,2332 \\
\hline Não & 40,4 & 42,1 & 35,6 & \\
\hline \multicolumn{5}{|l|}{ Declaração de uso de álcool pela vítima } \\
\hline Sim & 28,6 & 27,1 & 32,9 & 0,1965 \\
\hline Não & 71,4 & 72,9 & 67,1 & \\
\hline \multicolumn{5}{|l|}{ Evento relacionado ao trabalho } \\
\hline Sim & 8,2 & 6,9 & 11,8 & 0,0884 \\
\hline Não & 91,8 & 93,1 & 88,2 & \\
\hline \multicolumn{5}{|l|}{ Evento considerado acidental } \\
\hline Sim & 15,0 & 15,1 & 14,6 & 0,8822 \\
\hline Não & 85,0 & 84,9 & 85,4 & \\
\hline \multicolumn{5}{|l|}{ Atendimento prévio em outro serviço } \\
\hline Sim & 21,2 & 20,7 & 22,6 & 0,6189 \\
\hline Não & 78,8 & 79,3 & 77,5 & \\
\hline \multicolumn{5}{|l|}{ Período do atendimento } \\
\hline Diurno (07h00min-18h59min) & 44,0 & 44,7 & 42,1 & 0,5940 \\
\hline Noturno (19h00min-06h59min) & 56,0 & 55,3 & 57,9 & \\
\hline \multicolumn{5}{|l|}{ Dia do atendimento } \\
\hline Sábado e Domingo & 42,8 & 44,1 & 39,0 & 0,2682 \\
\hline Segunda a Sexta & 57,2 & 55,9 & 61,0 & \\
\hline \multicolumn{5}{|l|}{ Locomoção para o hospital } \\
\hline A pé/transporte coletivo & 24,6 & 23,6 & 27,5 & 0,1404 \\
\hline Veículo particular & 39,3 & 40,6 & 35,5 & \\
\hline Assistência pré-hospitalar ${ }^{\mathrm{d}}$ & 22,3 & 23,7 & 18,5 & \\
\hline Outros $^{e}$ & 13,8 & 12,1 & 18,5 & \\
\hline \multicolumn{5}{|l|}{ Natureza da lesão } \\
\hline Contusão/entorse/luxação & 30,0 & 32,1 & 23,3 & 0,2589 \\
\hline Corte/laceração/amputação & 44,0 & 42,3 & 49,2 & \\
\hline Fratura/trauma ${ }^{\mathrm{f}}$ & 12,7 & 12,7 & 12,5 & \\
\hline Outros $^{g}$ & 13,4 & 12,8 & 15,1 & \\
\hline \multicolumn{5}{|l|}{ Parte do corpo atingida } \\
\hline Cabeça/pescoço & 39,8 & 39,7 & 39,9 & 0,8735 \\
\hline Tórax/abdome/pélvis & 10,3 & 9,6 & 12,5 & \\
\hline Membros superiores & 24,9 & 25,7 & 22,2 & \\
\hline Membros inferiores & 8,2 & 8,1 & 8,4 & \\
\hline Múltiplos órgãos/regiões & 16,9 & 16,9 & 17,0 & \\
\hline \multicolumn{5}{|l|}{ Evolução ${ }^{\mathrm{h}}$} \\
\hline Alta & 79,3 & 80,8 & 74,5 & 0,2130 \\
\hline Internação hospitalar & 10,3 & 9,2 & 13,8 & \\
\hline Transferência para outro serviço & 10,4 & 10,0 & 11,7 & \\
\hline
\end{tabular}

Fonte: MS, SVS, Sistema de Vigilância de Violências e Acidentes-VIVA, Inquérito 2009. a) Para algumas variáveis o número de atendimentos divergiu devido a dados faltantes (ignorado/em branco). b) Teste do qui-quadrado. c) Inclui deficiência física, mental, visual, auditiva, outras deficiências/síndromes. d) Inclui unidades do Serviço de Atendimento Móvel de Urgência (SAMU), ambulâncias, resgate. e) Inclui viatura policial e outros. f) Inclui trauma cranioencefálico, trauma dentário, politraumatismo.g) Inclui intoxicação/envenenamento, queimadura, outros. h) Excluídos os casos que evoluíram ao óbito ( $\mathrm{n}=$ 8) nas primeiras 24 horas de atendimento. 
mais o SAMU ( $\mathrm{p}=0,0002)$. Não ha diferença estatisticamente significativa na natureza das lesões segundo faixa etária.

Os membros inferiores foram a parte do corpo mais atingida nos acidentes, enquanto lesões em múltiplos órgãos/regiões foram mais frequentes em mulheres de 20 a 39 anos ( $p=0,0013)$. Nas violências, a cabeça foi a parte do corpo mais atingida, sem diferença estatisticamente significativa entre as faixas etárias.

A alta foi o desfecho mais frequente nos casos de acidentes e violências, sem diferença entre as faixas etárias.
A Tabela 3 apresenta características dos atendimentos de emergência por acidentes. A queda foi o evento mais comum nas faixas etárias consideradas. Os acidentes de transporte (AT) foram mais frequentes em mulheres de 20 a 39 anos $(\mathrm{p}=0,0000)$. Considerando o tipo de queda, aquelas do mesmo nível predominaram em ambas as faixas etárias, entretanto a proporção foi maior entre as mulheres de 40 a 59 anos. As quedas de escada foram mais frequentes entre as mulheres de 20 a 39 anos $(\mathrm{p}=0,0000)$.

Dentre os AT, o passageiro foi o tipo de vítima mais frequente sendo maior em mulheres de 40 a

Tabela 3. Distribuição dos atendimentos de emergência por acidentes entre mulheres adultas segundo características dos eventos por faixa etária. 23 capitais e Distrito Federal, Brasil, 2009.

\begin{tabular}{|c|c|c|c|c|}
\hline \multirow[b]{2}{*}{ Características } & \multicolumn{3}{|c|}{ Faixa etária (anos) ${ }^{a}$} & \multirow[b]{2}{*}{ Valor de $p^{b}$} \\
\hline & $\begin{array}{c}\text { Total } \\
(\mathbf{n}) \\
\%\end{array}$ & $\begin{array}{c}20-39 \\
(\mathbf{n}) \\
\%\end{array}$ & $\begin{array}{c}40-59 \\
(\mathbf{n}) \\
\%\end{array}$ & \\
\hline Tipo de acidente & $(6.248)$ & $(4.004)$ & $(2.244)$ & \\
\hline Acidente de transporte & 26,3 & 31,5 & 17,7 & 0,0000 \\
\hline Queda & 38,6 & 33,1 & 47,8 & \\
\hline Queimadura & 2,9 & 3,1 & 2,6 & \\
\hline Outros acidentes & 32,2 & 32,3 & 31,9 & \\
\hline Tipo de vítima & $(1.818)$ & $(1.389)$ & $(429)$ & \\
\hline Pedestre & 15,3 & 13,9 & 19,5 & 0,0000 \\
\hline Condutor & 35,2 & 39,3 & 23,3 & \\
\hline Passageiro & 49,5 & 46,9 & 57,3 & \\
\hline Tipo de meio de locomoção & $(1.783)$ & $(1.364)$ & $(419)$ & \\
\hline A pé & 14,7 & 13,4 & 18,5 & 0,0000 \\
\hline Automóvel & 17,9 & 16,7 & 21,3 & \\
\hline Motocicleta & 44,9 & 50,9 & 26,7 & \\
\hline Bicicleta & 11,0 & 11,2 & 10,1 & \\
\hline Transporte coletivo & 11,7 & 7,8 & 23,3 & \\
\hline Tipo de queda & $(2.292)$ & $(1.254)$ & $(1.038)$ & \\
\hline Mesmo nível & 60,6 & 57,5 & 64,1 & 0,0025 \\
\hline Leito/mobília & 6,6 & 5,9 & 7,3 & \\
\hline Escada/degrau/árvore/telhado & 25,3 & 28,5 & 21,6 & \\
\hline Outros níveis ${ }^{c}$ & 7,6 & 8,1 & 7,0 & \\
\hline Tipo de queimadura & $(166)$ & $(108)$ & $(58)$ & \\
\hline Fogo/chama & 13,3 & 13,9 & 12,2 & 0,0742 \\
\hline Substância quente & 63,7 & 57,8 & 75,4 & \\
\hline Outros $^{\mathrm{d}}$ & 22,9 & 28,3 & 12,4 & \\
\hline Outros acidentes & $(1.863)$ & $(1.173)$ & $(690)$ & \\
\hline Ferimento cortante & 19,7 & 21,4 & 16,8 & 0,0209 \\
\hline Acidentes com animais & 13,2 & 11,5 & 16,0 & \\
\hline Queda de objeto sobre a pessoa & 10,9 & 11,4 & 10,2 & \\
\hline Choque contra objeto/pessoa & 15,2 & 14,2 & 16,8 & \\
\hline Entorse/esmagamento & 23,2 & 24,1 & 21,6 & \\
\hline Outros $^{e}$ & 17,9 & 17,4 & 18,7 & \\
\hline
\end{tabular}

Fonte: MS, SVS, Sistema de Vigilância de Violências e Acidentes-VIVA, Inquérito 2009. a) Para algumas variáveis o número de atendimentos divergiu devido a dados faltantes (ignorado/em branco). b) Teste do qui-quadrado. c) Inclui andaime e buraco. d) Inclui objeto quente, choque elétrico e substâncias químicas. e) Inclui sufocação/engasgamento, corpo estranho, afogamento, envenenamento/intoxicação, ferimento por arma de fogo, outros. 
59 anos $(p=0,0000)$. O meio de locomoção da vítima de AT com maior proporção de atendimentos foi a motocicleta, e maior proporção em mulheres jovens $(50,9 \%)$. As bicicletas responderam por $11 \%$ das ocorrências $(\mathrm{p}<0,0000)$. As queimaduras corresponderam a $2,9 \%$ dos eventos acidentais, sendo mais frequentes as causadas por substâncias quentes $(63,7 \%)$. Dentre os outros acidentes, destacaram-se as entorses e os esmagamentos $(23,2 \%)$ seguidos pelos ferimentos cortantes $(19,7 \%)$, que foram mais frequentes entre as mulheres de 20 a 39 anos ( $p=0,0209$ ).

As características dos atendimentos de emergência por violência podem ser vistas na Tabela 4. O tipo de violência mais comum foi a agressão $(84,6 \%)$, sem diferença estatisticamente significativa entre as faixas etárias. Observou-se maior percentual de agressor do sexo masculino $(79,1 \%)$ e identificado como parceiro íntimo $(44,1 \%)$, porém sem diferença estatisticamente significativa entre as faixas etárias.
A violência foi predominantemente física, com maior proporção em mulheres de 20 a 39 anos $(98,9 \% ; p=0,0059)$. A violência sexual foi relatada em 2,9\% das ocorrências, sendo mais frequente em mulheres de 40 a 59 anos $(6,2 \% ; p=0,0271)$. A violência psicológica ocorreu em $41 \%$ dos casos, sendo concomitante a outros tipos de violência e a negligencia em 1,1\%.

\section{Discussão}

Os resultados encontrados para mulheres adultas (20 a 59 anos) participantes do Inquérito VIVA 2009 mostram predomínio do atendimento de emergência para as vítimas de acidentes.

A maior proporção dos acidentes foi considerada acidental sendo necessário aprofundamento na análise dos $6,7 \%$ considerados não acidentais, para melhor compreensão da sua ocorrência.

Tabela 4. Distribuição dos atendimentos de emergência por violência entre mulheres adultas segundo características dos eventos por faixa etária. 23 capitais e Distrito Federal, Brasil, 2009.

\begin{tabular}{|c|c|c|c|c|}
\hline \multirow[b]{2}{*}{ Características } & \multicolumn{3}{|c|}{ Faixa etária (anos) ${ }^{a}$} & \multirow[b]{2}{*}{ Valor de $\mathrm{p}^{\mathrm{b}}$} \\
\hline & $\begin{array}{c}\text { Total } \\
(\mathbf{n}) \\
\%\end{array}$ & $\begin{array}{c}20-39 \\
(\mathbf{n}) \\
\%\end{array}$ & $\begin{array}{c}40-59 \\
(\mathbf{n}) \\
\%\end{array}$ & \\
\hline Tipo de violência & $(717)$ & $(555)$ & $(162)$ & \\
\hline Violência autoprovocada & 15,5 & 15,2 & 16,3 & 0,7569 \\
\hline $\begin{array}{l}\text { Violência interpessoal (agressão, } \\
\text { maus tratos e intervenção legal) }\end{array}$ & 84,6 & 84,8 & 83,7 & \\
\hline Agressor $^{\mathrm{d}}$ & $(568)$ & $(444)$ & $(124)$ & \\
\hline Parceiroíntimo ${ }^{\mathrm{e}}$ & 44,1 & 44,9 & 41,7 & 0,6301 \\
\hline Conhecido $^{\mathrm{f}}$ & 33,8 & 32,5 & 37,6 & \\
\hline Desconhecidog & 22,1 & 22,5 & 20,7 & \\
\hline Sexo do agressor ${ }^{\mathrm{d}}$ & $(545)$ & $(427)$ & $(118)$ & \\
\hline Masculino & 79,1 & 79,3 & 78,1 & 0,7956 \\
\hline Feminino & 21,0 & 20,7 & 21,9 & \\
\hline Violência física & $(580)$ & $(452)$ & $(128)$ & \\
\hline Sim & 97,9 & 98,9 & 94,8 & 0,0059 \\
\hline Não & 2,1 & 1,1 & 5,2 & \\
\hline Violência sexual & $(550)$ & $(429)$ & $(121)$ & \\
\hline Sim & 2,9 & 1,8 & 6,2 & 0,0271 \\
\hline Não & 97,1 & 98,2 & 93,9 & \\
\hline Violência psicológica & $(556)$ & $(432)$ & $(124)$ & \\
\hline Sim & 41,0 & 41,3 & 40,2 & 0,8356 \\
\hline Não & 59,0 & 58,7 & 59,8 & \\
\hline Negligência & $(549)$ & $(429)$ & $(120)$ & \\
\hline Sim & 1,1 & 1,0 & 1,1 & 0,9439 \\
\hline Não & 98,9 & 99,0 & 98,9 & \\
\hline
\end{tabular}

Fonte: MS, SVS, Sistema de Vigilância de Violências e Acidentes-VIVA, Inquérito 2009. a) Para algumas variáveis o número de atendimentos divergiu devido a dados faltantes (ignorado/em branco). b) Teste do qui-quadrado. c) Inclui maus-tratos e intervenção por agente público legal. d) Refere-se apenas aos casos de agressão. e) Inclui (ex-)marido, (ex-)namorado. f) Inclui pai/mãe, outros familiar, amigo/conhecido. g) Inclui agente público legal. 
As características das vítimas de acidentes de transporte (AT) incluídas nesse estudo são similares às descritas em outros estudos de AT no país, com maior proporção de mulheres na faixa etária de 20 a 39 anos $^{12}$, negras (preta e parda) e com escolaridade igual ou maior que 9 anos de estudo $^{13}$. Existem poucos estudos na literatura que tracem o perfil dessas mulheres, pois a ocorrência de AT é historicamente maior em homens.

Entre os AT, o passageiro foi o tipo de vítima mais comum. Resultado semelhante foi encontrado em estudo realizado em Maringá com mulheres vítimas de AT onde mulheres jovens, na faixa até 20 anos, se envolveram em AT principalmente como passageiras ou pedestres ${ }^{13}$.

A tendência crescente dos AT envolvendo motocicletas é observada em vários países. No Brasil, inúmeros estudos relatam maior percentual de vítimas de AT ocupantes de motocicle$\mathrm{ta}^{14,15}$. No presente estudo, o encontro da motocicleta como principal meio de locomoção e do passageiro como principal tipo de vítima pode estar relacionada ao seu uso como meio de transporte por mulheres, mas na condição de passageira. Segundo estudo desenvolvido por Andrade e Jorge ${ }^{16}$ em Londrina, Paraná, as mulheres se acidentaram mais como passageiras de motocicletas $(51,8 \%)$.

Certamente, está relacionado também ao uso de motocicletas o encontro de maior proporção de membros inferiores como parte do corpo mais atingida e predomínio de contusões/entorses/luxação como natureza da lesão. Estes resultados são semelhantes aos observados em outros estudos e podem estar relacionados à obrigatoriedade do uso do capacete no país inclusive pelos passageiros o que proporciona maior proteção da cabeça e pescoço ${ }^{12,17}$.

$\mathrm{O}$ achado de queda como tipo de acidente mais frequente entre as mulheres no Inquérito VIVA 2009 pode também estar relacionado à contusões/entorses/luxação, natureza da lesão encontrada com maior frequência nesse estudo.

Apesar de a violência se apresentar com frequência menor que os acidentes nesse estudo, é um evento intencional que traz grandes desafios para a área da saúde na sua abordagem e superação. Minayo ${ }^{18}$ discute em artigo que aborda a inclusão da violência na agenda da Saúde que $a$ maior dificuldade está em convencer um setor profundamente marcado pela racionalidade biomédica em aceitar nesse modelo e dinâmica complexos problemas de caráter social e não doenças.

Nas mulheres vítimas de violência observouse maior proporção de atendimentos na faixa etária de 20 a 39 anos, negras (preta/parda) e com menor escolaridade ( 0 a 8 anos de estudo). Essas mesmas características foram relatadas em estudo desenvolvido na cidade de Recife ${ }^{19}$.

O tipo de violência com maior percentual de ocorrência foi a interpessoal (agressão, maus tratos e intervenção legal), com suas diversas formas de expressão. A violência física foi predominante, mas com alto percentual de violência psicológica. $\mathrm{O}$ agressor mais frequente foi o parceiro íntimo com a violência acontecendo no domicílio. Estudo sobre violência contra a mulher por parceiro íntimo em regiões do Brasil encontrou a violência psicológica como o evento mais frequente na vida e no ano anterior à pesquisa. Encontrou ainda que a violência psicológica acompanhou em $90 \%$ das vezes os relatos das agressões físicas ou sexuais ${ }^{20}$.

$\mathrm{Na}$ violência em que a mulher é a vítima, observa-se ainda um grande domínio do agressor do sexo masculino. As relações sociais desiguais, associando padrões de comportamento de maior agressividade, desigualdade de poder e força, inscrevem a violência de gênero como mecanismo de controle nas relações pessoais e familiares. A violência pode representar comportamento de reconquista de poder ou forma usada pelo homem para prevenir sua perda ${ }^{6}$.

Especificamente nos casos de violência de gênero, a demanda para que os serviços de saúde/ rede de saúde registrem de forma apurada, estejam preparados para identificar os casos e para a especificidade da atenção, acompanha o crescimento da exigência de políticas públicas de prevenção, promoção, proteção e atenção à violência de gênero no país. A extensão da rede de saúde a torna uma porta de acesso privilegiado para as mulheres em situação de violência. Assim, é cada vez mais relevante que os serviços estejam capacitados para a atenção integral e humanizada às vítimas e para o desenvolvimento de ações de prevenção e promoção frente a um problema que, muitas vezes, não é explicitado de maneira clara pela vítima ${ }^{21}$. O reconhecimento de que a casa, o ambiente doméstico é um local de frequente exercício da violência contra as mulheres, também se enfrenta com uma queda de parâmetros culturais e sociais que exigem uma perspectiva própria nas políticas públicas. É recorrente nas pesquisas a indicação de que, quando a vítima é mulher, o agressor é na grande maioria dos casos do sexo masculino, parceiro íntimo (incluindo maridos, companheiros, namorados ou exparceiros) ou membros da família ${ }^{22}$. Essa dinâmica das relações sociais de sexo, que engendram 
relações de poder expressas no exercício da violência, torna a resposta ao problema mais complexa, exigindo uma integração de distintas áreas de atendimento e uma integração efetiva dos serviços públicos ${ }^{21}$

A associação do álcool à ocorrência de acidentes e violências está relatada na literatura ${ }^{4,23,24}$. Por ser droga socialmente aceita, o álcool é mais largamente consumido produzindo alterações neuromotoras e de comportamento em diferentes concentrações. Os acidentes, principalmente de trânsito, associados ao uso do álcool precisam ser vistos como totalmente preveníveis e evitáveis, perdendo o caráter de casualidade que muitas vezes lhe é imputado. Em relação à violência, também se relata a associação entre o consumo de álcool podendo levar a mudanças de humor e comportamento, favorecendo a ocorrência de atos violentos.

Estudos realizados a partir dos dados do Inquérito VIVA contribuem para o conhecimento e o dimensionamento da ocorrência de acidentes e violências na população geral e em grupos específicos, como neste recorte de gênero. Assim, ganham visibilidade contribuindo na discussão e proposição de políticas públicas para a prevenção das causas externas, principalmente a violência, ainda muito oculta e que não pode e não deve ser vista e considerada evento tão irremediável como faz parecer. Não deve ser aceita como um aspecto inevitável da condição humana. A violência contra as mulheres, assim como os acidentes, podem ser evitados e ter suas consequências reduzidas no país.

\section{Colaboradores}

CS Rodrigues, DC Malta, T Godinho, MDM Mascarenhas, MMA Silva e RE Silva participaram igualmente de todas as etapas de elaboração do manuscrito e aprovaram a versão final do texto. 


\section{Referências}

1. Mascarenhas MDM, Monteiro AP, Sá NNB, Gonzaga LAA, Neves ACM, Roza DL, Silva MMA, Duarte EC, Malta DC. Epidemiologia das causas externas no Brasil: mortalidade por acidentes e violências no período de 2000 a 2009. In: Brasil. Ministério da Saúde (MS). Saúde Brasil 2010: uma análise da situação de saúde e de evidências selecionadas de impacto de ações de vigilância em saúde. Brasília: MS; 2011. p. 226-247.

2. Brasil. Portaria GM n $737 / 2001$, de 16 de maio de 2001. Aprova a Política Nacional de Redução da Morbimortalidade por Acidentes e Violências. Diário Oficial da União 2001; 17 maio.

3. Brasil. Portaria GM no $1256 / 2006$, de 23 de junho de 2006. Institui incentivo aos estados, ao Distrito Federal e aos municípios para a Vigilância de Acidentes e Violências em Serviço Sentinela. Diário Oficial da União 2006; 24 jun.

4. Toledo AG, Chagas DMM, Agripini Filho D. O Consumo de álcool e os acidentes de trânsito - Pesquisa sobre a associação entre consumo de álcool e os acidentados de trânsito nas cinco regiões brasileiras. Recife: CCS Gráfica e Editora; 2011.

5. Rodrigues CS, Ishitani LH, Almeida MCM, Evangelista PA, Ladeira RM. Qualificação das informações sobre acidentes de trânsito em BH: subsídios às ações de intervenção. In: Magalhães Júnior HM, organizador. Desafios e inovações na gestão do SUS em Belo Horizonte: a experiência de 2003 a 2008. Belo Horizonte: Mazza Edições; 2010. p. 485-498.

6. Schraiber LB, D'Oliveira AFPL, Couto MT. Violência e saúde: contribuições teóricas, metodológicas e éticas de estudos da violência contra a mulher. Cad Saude Publica 2009; 25(Supl. 2):5205-5216.

7. Ministério da Saúde (MS). Viva: vigilância de violências e acidentes, 2006 e 2007. Brasília: MS; 2010.

8. Brasil. Ministério da Saúde (MS). Viva: vigilância de violências e acidentes, 2008 e 2009. Brasília: MS; 2011.

9. World Health Organization (WHO), London School of Hygiene and Tropical Medicine. Preventing intimate partner and sexual violence against women: taking action and generating evidence. Geneva: WHO; 2010.

10. Brasil. Ministério da Saúde (MS). Conselho Nacional de Saúde. Resolução nº. 196/1996. Diretrizes e Normas Regulamentadoras de Pesquisas Envolvendo Seres Humanos. Diário Oficial da União 1996; out 10 .

11. StataCorp. Stata statistical software: release 10. College Station. TX: StataCorp LP; 2007.

12. GawryszewskiI VP, Coelho HMM, Scarpelini S, Zan R, Jorge MHPM, Rodrigues EMS. Perfil dos atendimentos a acidentes de transporte terrestre por serviços de emergência em São Paulo, 2005. Rev Saude Publica 2009; 43(2):275-282.

13. Davantel PP, Pelloso SM, Carvalho, MDB, Oliveira NLB. A mulher e o acidente de trânsito: caracterização do evento em Maringá, Paraná. Rev Bras Epidemiol 2009; 12(3):355-367.
14. Ladeira RM, Barreto SM. Fatores associados ao uso de serviço de atenção pré-hospitalar por vítimas de acidentes de trânsito. Cad Saude Publica 2008; 24(2):287-294

15. Marín-Léon L, Belon AP, Barros MBA, Almeida SDM, Restitutti MC. Tendência dos acidentes de trânsito em Campinas, São Paulo, Brasil: importância crescente dos motociclistas. Cad Saude Publica 2012; 28(1):39-51.

16. Andrade SM, Jorge MHPM. Características das vítimas por acidentes de transporte terrestre em município da Região Sul do Brasil. Rev Saude Publica 2000; 34(2):149-156.

17. Parreira JG, Gregorut F, Perlingeiro JAG, Solda SC, Asser JC. Análise comparativaentre as lesões encontradas em motociclistas envolvidos em acidentes de trânsito e vítimas de outros mecanismos de trauma fechado. Rev Assoc Med Bras 2012; 58(1):76-81.

18. Minayo MCS. The inclusion of violence in the health agenda: historical trajectory. Cien Saude Colet 2006; 11(2):357-383

19. Silva MA, Neto GHF, Figueiroa JN, Filho JEC. Violence against women: prevalence and associated factors in patients attending a public healthcare service in the Northeast of Brazil. Cad Saude Publica 2010; 26(2):264-272.

20. Schraiber LB, D’Oliveira AFPL, França-Júnior I, Diniz S, Portella AP, Ludermir AB, Couto MT. Prevalência da violência contra a mulher por parceiro íntimo em regiões do Brasil. Rev Saude Publica 2007; 41(5):797-807.

21. Schraiber LB, D’Oliveira AFPL, França Júnior I, Strake SS, Oliveira EEA. A violência contra as mulheres: demandas espontâneas e busca ativa em unidade básica de saúde. Saúde e sociedade 2000; 9(1/2):3-15.

22. Schraiber LB, Bandeira L, Almeida TMC. Desafios das políticas e ações em saúde diante da violência contra as mulheres. SER Social 2008; 10(22):183-212.

23. Malta DC, Filho AMS, Montenegro MMS, Mascarenhas MDM, Silva MMA, Lima CM, Morais Neto OL, Temporão JG, Penna GO. Análise da mortalidade por acidentes de transporte terrestre antes e após a Lei Seca - Brasil, 2007-2009. Epidemiol. Serv. Saúde 2010; 19(4):317-328.

24. Rabello PM, Caldas Júnior AF. Violência contra a mulher, coesão familiar e drogas. Rev Saude Publica 2007; 41(6):970-978.

Artigo apresentado em 10/06/2012

Aprovado em 04/07/2012

Versão final apresentada em 14/07/2012 